Social-choice self-administration chamber

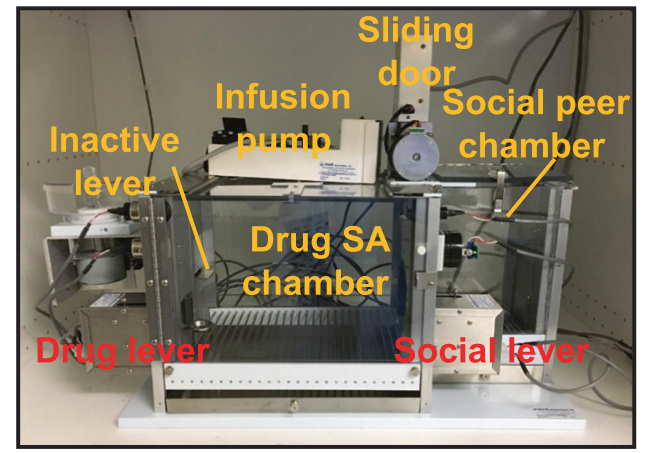

Fig. 1 Custom-made social-choice self-administration apparatus. For instructions on how to build the apparatus, see [6], and technical advice is available upon request from the authors

demonstrated the generality of food-choice voluntary abstinence to female rats and to heroin, and that surprisingly voluntary abstinence prevents incubation of heroin craving [3]. In mechanistic studies, we found a role of dorsomedial striatum neuronal ensembles in incubation of methamphetamine craving after voluntary abstinence [4], and a role of glutamatergic projections from the anterior insular cortex to central amygdala in relapse after voluntary abstinence [5].

However, the use of palatable food as the nondrug reward may limit the model's clinical translation. This is because for most humans, the rewards that compete with drugs are primarily social (family and employment). Based on this consideration, we recently introduced a newer voluntary abstinence model that involves choices between a drug and operant access to social interaction [6] (Fig. 1). We found that rats trained in established addiction models-escalation, DSM-IV-based, and intermittent access-will voluntarily abstain when given mutually exclusive choices between methamphetamine or heroin versus social interaction. This effect was independent of their 'addiction score', persisted through 4 weeks of forced abstinence, and could only be reversed by delay or punishment of the social reward. We also found that social-choice-induced voluntary abstinence prevents the emergence of incubation of methamphetamine craving, even 1 month after cessation of the social choice. This protective effect was associated with activation (assessed by the activity marker Fos) of inhibitory central amygdala PKC $\delta$-expressing neurons and decreased neuronal activity in the anterior insular cortex [6].

In conclusion, we introduced two novel models of choice-based voluntary abstinence and demonstrated the profound protective effects of positive social interaction on drug addiction and relapse in rat models. Our findings support wider implementation of social-based behavioral treatments, which include not only the established community reinforcement approach, but also socialbased psychotherapies and family-based social support systems to provide social support before and during drug-seeking episodes.

\section{ACKNOWLEDGEMENTS}

The research was supported by the Intramural Research Program of NIDA (YS), NARSAD Distinguished Investigator Grant Award (YS), and a fellowship from the NIH Center on Compulsive Behaviors (MV).Competing interests: The authors declare no competing interests

Publisher's note: Springer Nature remains neutral with regard to jurisdictional claims in published maps and institutional affiliations.

\section{REFERENCES}

1. Venniro M, Caprioli D, Shaham Y. Animal models of drug relapse and craving: from drug priming-induced reinstatement to incubation of craving after voluntary abstinence. Prog Brain Res. 2016;224:25-52.

2. Caprioli D, Venniro M, Zeric T, Li X, Adhikary S, Madangopal R, et al. Effect of the novel positive allosteric modulator of metabotropic glutamate receptor 2 AZD8529 on incubation of methamphetamine craving after prolonged voluntary abstinence in a rat model. Biol Psychiatry. 2015;78:463-73.

3. Venniro M, Zhang M, Shaham Y, Caprioli D. Incubation of methamphetamine but not heroin craving after voluntary abstinence in male and female rats. Neuropsychopharmacology. 2017;42:1126-35.

4. Caprioli D, Venniro M, Zhang M, Bossert JM, Warren BL, Hope BT, Shaham Y. Role of dorsomedial striatum neuronal ensembles in incubation of methamphetamine craving after voluntary abstinence. J Neurosci. 2017;37:1014-27.

5. Venniro M, Caprioli D, Zhang M, Whitaker LR, Zhang S, Warren BL, et al. The anterior insular cortex-->central amygdala glutamatergic pathway is critical to relapse after contingency management. Neuron. 2017;96:414-27 e418.

6. Venniro M, Zhang M, Caprioli D, Hoots JK, Golden SA, Heins C, et al. Volitional social interaction prevents drug addiction in rat models. Nature Neuroscience. 2018 (In press).

\title{
An emerging epigenetic framework of systemic and central mechanisms underlying stress-related disorders
}

Carla Nasca ${ }^{1}$, Natalie Rasgon ${ }^{1,2}$ and Bruce McEwen ${ }^{1}$

Neuropsychopharmacology (2019) 44:235-236; https://doi.org/10.1038/s41386-018-0227-1

Advances in translational neuroscience are pointing to a new paradigm for conceptualizing diagnosis and treatment of major morbidities, such as major depressive disorders (MDD), diabetes, and dementia [1]. Here we explore the emerging framework that focuses on the epigenetic actions of metabolic mediators on regulation of gene expression in brain regions controlling cognition and emotion as an approach to examine the systemic, as well as neural bases, of stress-related CNS disorders.

\footnotetext{
${ }^{1}$ Harold and Margaret Milliken Hatch Laboratory of Neuroendocrinology, The Rockefeller University, New York, USA and ${ }^{2}$ Center for Neuroscience in Women's Health, Stanford University, Palo Alto, CA 91304, USA

Correspondence: Carla Nasca (cnasca@rockefeller.edu) or Bruce McEwen (mcewen@rockefeller.edu)
}

Published online: 7 October 2018 
Central to this epigenetic framework linking neural and systemic functions is the concept of allostasis (adaptation) and allostatic load (pathophysiology) [1]. This concept emphasizes that endogenous mediators for adaptation contribute to pathologies, including MDD, when activated persistently or dysregulated under circumstances of toxic stress and associated health-damaging behaviors [1]. There is an increasing scientific consensus to support a connection between systemic illness and MDD, a disorder still primarily seen as brain based. Recently, we reported decreased plasma levels of acetyl-L-carnitine (LAC) in two cohorts of patients suffering with MDD [2]. The LAC deficiency was greater with stronger severity, earlier disease onset and treatment-resistant-depression, which was associated with childhood trauma [2]. In rodent models, targeting the deficiency in LAC by supplementation of exogenous LAC leads to antidepressant-like responses after a few days of administration, while standard antidepressants require multiple weeks [3-5]. LAC is an endogenous molecule with a plethora of biological functions important for brain plasticity and systemic metabolism relevant to stress and pathophysiology of MDD [3]. In rodent models with sustained activation of neural mineralocorticoid receptors (MR), supplementation of LAC increased acetylation of histone markers of activate gene transcription leading to increased expression of an inhibitor of spontaneous glutamate release, mGluR2, and the neurotrophin, BDNF $[3,4]$. Normalizing glutamate overflow and BDNF expression ameliorated decreased dendritic branching in the hippocampus and connected brain areas important for the pathophysiology of depression [1, 3-6].

Within this framework, it is important to emphasize that, at least in rodent models, low $L A C$ serves as a biomarker of insulin resistance (IR), which was also ameliorated by supplementation with LAC [4]. IR is a modifiable inflammatory state that is often observed in patients suffering from MDD [1]. Furthermore biomarkers of allostatic load involving imbalance in systemic stress-related physiology, and also including heightened release of proinflammatory cytokines and hypercortisolemia [1], are inversely correlated with hippocampal volume in patients with affective dysregulation [1].
These studies provide a foundation for future research of the mechanisms of LAC action related to the pathophysiological role of systemic physiology in a variety of stress-related CNS disorders, including MDD and interrelated disorders, such as Alzheimer's disease. This framework can lead to identify therapeutic targets for development of mechanism-based treatment strategies tailored to biologically defined patient populations [5].

\section{FUNDING}

Authors acknowledge funding support from the American Foundation for Suicide Prevention (AFSP), the Robertson Foundation and the Hope for Depression Research Foundation (HDRF) (to C.N. and B.S.M.).Competing interests: The authors declare no competing interests.

Publisher's note: Springer Nature remains neutral with regard to jurisdictional claims in published maps and institutional affiliations.

\section{REFERENCES}

1. Rasgon NL, McEwen BS. Insulin resistance-a missing link no more. Mol Psychiatry. 2016;21:1648-52.

2. Nasca C, Bigio B, Lee FS, Young SP, Kautz MM, Albright A, et al. Acetyl-I-carnitine deficiency in patients with major depressive disorder. Proceedings of the National Academy of Sciences. 2018;115:8627-32.

3. Nasca $C$, Xenos $D$, Barone $Y$, Caruso A, Scaccianoce $S$, Matrisciano $F$, et al. L-acetylcarnitine causes rapid antidepressant effects through the epigenetic induction of mGlu2 receptors. Proc Natl Acad Sci USA. 2013;110:4804-9.

4. Bigio B, Mathe AA, Sousa VC, Zelli D, Svenningsson P, McEwen BS, et al. Epigenetics and energetics in ventral hippocampus mediate rapid antidepressant action: Implications for treatment resistance. Proc Natl Acad Sci USA. 2016;113:7906-11.

5. Nasca C, Bigio B, Zelli D, de Angelis $P$, Lau T, Okamoto $M$, et al. Role of the astroglial glutamate exchanger $\mathrm{xCT}$ in ventral hippocampus in resilience to stress. Neuron. 2017;96:402-13. e405

6. Akil H, Gordon J, Hen R, Javitch J, Mayberg H, McEwen B, et al. Treatment resistant depression: a multi-scale, systems biology approach. Neurosci Biobehav Rev. 2017; 84:272-88. 